\title{
Application of a Poisson-gamma model to study the influence of gamete concentration on sperm-oocyte fusion in the zona-free hamster egg penetration test
}

\author{
R. J. Aitken and R. A. Elton* \\ $M R C$ Unit of Reproductive Biology, Centre for Reproductive Biology, 37 Chalmers Street, \\ Edinburgh EH3 $9 E W$, and ${ }^{*}$ Medical Computing and Statistics Unit, University of Edinburgh, \\ Edinburgh EH8 $9 A G, U . K$.
}

\begin{abstract}
Summary. A Poisson-gamma distribution model has been developed for studying sperm-oocyte interaction in the zona-free hamster egg penetration test. With the aid of this model we have analysed the relationship between the penetration results achieved with semen of normal fertile men and the concentration of spermatozoa and oocytes used in the assay. From this analysis we have drawn up reference tables estimating the number of spermatozoa and occytes which must be present in the assay for the result to represent a significant $(P<0.05)$ decline in sperm function relative to the normal fertile population.

Since there is, as yet, no fixed protocol for the penetration assay, these tables have been constructed to fit a range of methodologies varying with respect to the nature of the preincubation phase and including (a) a 7-h preincubation in Medium BWW, (b) a 3-h preincubation in hyperosmotic Medium BWW or (c) a 3-h preincubation in the presence of A23187.
\end{abstract}

\section{Introduction}

The zona-free hamster egg penetration test (Yanagimachi et al., 1976) provides a measure of the ability of human spermatozoa to capacitate, acrosome react and generate a fusogenic equatorial segment competent to fuse with the vitelline membrane of the oocyte. The clinical usefulness of the test as a diagnostic procedure has been suggested by retrospective (Rogers et al., 1979; Rogers, 1985) and prospective (Aitken et al., 1984a) studies, particularly when the results are combined with data describing the movement characteristics of the spermatozoa (Aitken et al., 1984a; Aitken, 1985). However, the test does not examine every aspect of sperm function required to initiate a pregnancy and, for this reason, positive results cannot be taken as conclusive evidence of normal fertility. The diagnostic value of the test therefore rests heavily with negative or poor levels of penetration and the assumption that, if sperm-oocyte fusion cannot occur in this heterologous system, it will also fail when a human oocyte is involved.

However, serious difficulties of interpretation arise when the test is applied to cases of severe oligozoospermia, as will frequently be the case in the analysis of idiopathic male infertility (Aitken, 1985 ) or the evaluation of new approaches to male contraception. Since the concentration of motile spermatozoa employed in the assay influences the penetration rates observed (Aitken et al., 1982) it is possible that, in such cases, low levels of fusion may be observed as a consequence of inadequate sperm numbers, rather than any defect in the penetrating potential of the spermatozoa. Hence, guidelines are clearly required to indicate the minimal number of spermatozoa and oocytes which should be present in the assay for a poor result to be indicative of defective sperm function and not simply a reflection of an insufficient concentration of gametes. 
We have recently described the use of Poisson distribution theory to analyse the relationship between motile sperm concentration and the level of penetration observed with zona-free hamster eggs (Aitken \& Elton, 1984, 1986). In this paper we extend these studies to investigate the interaction between sperm concentration, oocyte number and penetration rate observed for populations of normal fertile men. Using a Poisson-gamma probability model we have examined the quantitative nature of sperm-oocyte interaction for 3 different versions of this assay system and constructed reference tables, indicating the threshold numbers of spermatozoa and oocytes which must be exceeded if a negative or poor result is to be taken as indicative of a statistically significant reduction of sperm function.

\section{Materials and Methods}

The donors were recruited from a pre-vasectomy clinic, were all of proven fertility and exhibited the semen profiles presented in Table 1 . Semen samples were produced by masturbation, after at least $48 \mathrm{~h}$ abstinence, and were left for at least $30 \mathrm{~min}$ for liquefaction to occur. The liquefied semen was subjected to a conventional semen analysis (World Health Organization, 1980) after which the spermatozoa were washed free of seminal plasma by repeated $(\times 3)$ cycles of centrifugation $(5 \mathrm{~min}$ at $500 \mathrm{~g}$ ) and resuspension in medium BWW (Biggers et al., 1971). The performance of the spermatozoa in the zona-free hamster egg assay was examined at a total sperm concentration of $10 \times 10^{6}$ spermatozoa/ml using 3 different protocols incorporating preincubation phases of $7 \mathrm{~h}$ in normosmotic Medium BWW (Aitken et al., 1982), $3 \mathrm{~h}$ in hyperosmotic Medium BWW (Aitken et al., 1983) or $3 \mathrm{~h}$ in the presence of $\mathrm{A} 23187$ prepared as an aqueous suspension of the $\mathrm{Ca}^{2+} / \mathrm{Mg}^{2+}$ salt as described by Aitken et al. (1984b). These incubations were carried out in loosely capped sterile centrifuge tubes (Sterilin, Feltham, Middlesex, U.K.) at $37^{\circ} \mathrm{C}$ in an atmosphere of $5 \% \mathrm{CO}_{2}$ in air. At the end of the preincubation phase the spermatozoa were centrifuged at $500 \mathrm{~g}$ for $5 \mathrm{~min}$, resuspended in normal Medium BWW and then analysed to determine the concentration of motile cells. The spermatozoa were distributed as $50 \mu \mathrm{l}$ droplets under liquid paraffin in plastic culture dishes (Falcon 3002, Oxnard, CA, U.S.A.) and incubated for a further $3 \mathrm{~h}$ at $37^{\circ} \mathrm{C}$ in an atmosphere of $5 \% \mathrm{CO}_{2}$ in air. At the end of this incubation phase the oocytes were washed free of loosely adherent spermatozoa, mounted on glass slides and compressed to a depth of about $30 \mu \mathrm{m}$ before examination by phase-contrast microscopy for swollen sperm heads with an attached or closely associated tail.

Table 1. Semen profiles of donors used to establish values presented in Tables 2 and 3 (mean \pm s.e.m.)

\begin{tabular}{|c|c|c|c|c|c|}
\hline Technique & No. of men & $\begin{array}{c}\text { Sperm } \\
\text { concentration } \\
\left(10^{-6} / \mathrm{ml}\right)\end{array}$ & $\begin{array}{l}\text { Sperm } \\
\text { motility } \\
(\%)\end{array}$ & $\begin{array}{c}\text { Normal } \\
\text { morphology } \\
(\%)\end{array}$ & $\begin{array}{c}\text { Semen } \\
\text { volume } \\
(\mathrm{ml})\end{array}$ \\
\hline Conventi & 26 & $117 \cdot 2 \pm 17 \cdot 6$ & $55 \cdot 7 \pm 1.9$ & $53 \cdot 6 \pm 1 \cdot 7$ & $2 \cdot 8 \pm 0 \cdot 4$ \\
\hline Hyperosmotic $\uparrow$ & 42 & $134.8 \pm 13.4$ & $62 \cdot 1 \pm 1 \cdot 6$ & $54.0 \pm 1.7$ & $3.5 \pm 0.2$ \\
\hline A23187t & 55 & $113.8 \pm 13.3$ & $63.9 \pm 1.7$ & $56.9 \pm 1.9$ & $2.9 \pm 0.2$ \\
\hline
\end{tabular}

*Aitken et al. (1982).

$\dagger$ Aitken et al. (1983).

$\ddagger$ Aitken et al. (1984b); aqueous suspension of $\mathrm{Ca}^{2+} / \mathrm{Mg}^{2+}$ salt at $100 \mu \mathrm{M}(0.05 \mathrm{mg} / \mathrm{ml})$.

\section{The Poisson-gamma model}

The notation of our previous studies is used (Aitken \& Elton, 1984, 1986) in which an experiment involving $n$ oocytes and a sperm sample with a motile sperm density of $x \times 10^{6} / \mathrm{ml}$ 
results in a mean penetration rate of $m$ spermatozoa per oocyte. In a previous analysis (Aitken $\&$ Elton, 1984) we demonstrated that data generated by the hamster egg penetration test is well fitted by a model in which the total number of penetrating spermatozoa $(t=n m)$ has a Poisson distribution with parameter $n \theta x$. Hence the level of penetration observed is influenced by the number of oocytes used in the assay $(n)$, the concentration of motile spermatozoa $(x)$ and the unknown factor, $\theta$, which represents the functional competence of the sperm population and can be thought of as the mean number of spermatozoa penetrating each oocyte at a concentration of $1 \times 10^{6}$ motile spermatozoa/ml.

Within a population of normal fertile men there will be variation in the value of $\theta$ between individuals. Thus the variability in the values of $m$ observed, even after correcting for differences in gamete concentration, will be much greater than expected from a Poisson model with constant $\theta$. We have therefore to extend the model to incorporate a distribution of different $\theta$ values among the normal fertile population, and an appropriate choice from a mathematical viewpoint is the gamma distribution, with probability density given by

$$
\mathbf{P}(\theta)=\alpha^{r} \theta^{r-1} \exp (-\alpha \theta) / \Gamma(r),
$$

where $\Gamma$ represents the gamma function. This model is found, empirically, to give a satisfactory fit to real data, both in the present paper as well as in a recent study of asthenozoospermic patients (Aitken \& Elton, 1986). The distribution of observed values of $t$ for fixed $n x$ generated by the Poisson and gamma models is a negative binomial (Johnson \& Kotz, 1969). Maximum likelihood (ML) methods (Mather, 1964) can be used to estimate the parameters $\alpha$ and $r$, in the above equation, and hence derive the mean value $(r / \alpha)$ and variance $\left(r / \alpha^{2}\right)$ of the distribution of $\theta$ in the group of men concerned.

Once the distribution of $\theta$ has been characterized for the normal fertile population, maximum likelihood methods can be used to give likelihood ratio tests for comparing the above parameter values with those recorded for other groups of men (Aitken \& Elton, 1986; R. A. Elton \& R. J. Aitken, unpublished observations). The model can also be applied to the testing of individual sperm samples against a data base of results obtained for a defined population, in this case the normal fertile population. Using the negative binomial model, the probability $q(t)$ of observing $t$ spermatozoa in a further individual sampled from the same group can be expressed as: $r(r+1) \ldots$ $(r+t-1)(n x)^{t} \alpha^{r} /\left[(n x+\alpha)^{t+r} t !\right]$ where $n$ and $x$ are the number of oocytes and motile sperm concentration $\left(10^{6} / \mathrm{ml}\right)$ for that individual. Thus the probability of observing a particular value of $t$ for a semen sample from a defined population with a characterized distribution for $\theta$, and hence known $r$ and $\alpha$ values, depends only on the product $(n x)$ of the number of oocytes and spermatozoa used in carrying out the test. In particular

$$
q(0)=\alpha^{r} /(n x+\alpha)^{r}
$$

This formula can be inverted to give

$$
n x=\alpha(1 / \sqrt[r]{q(0)}-1)
$$

allowing calculation of the number of gametes required to give a specified low probability (such as $P=0.05$ ) of observing no sperm penetration at all in the experiment. More complex calculations (using numerical methods) can be used to set corresponding lower limits for observing as few as 1,2 or 3 penetrating spermatozoa in an experiment. In this way a table of limits may be drawn up, for the normal fertile population, of the gamete numbers $(n x)$ which would be expected to give only a $5 \%$ probability of generating a specific level of penetration when the functional competence of the spermatozoa $(\theta)$ was within the normal range. Providing that for any given level of penetration $(t=0,1,2$ etc) the value for $n x$ in such a table is exceeded, then significant evidence of defective sperm function can be said to exist. If $n x$ is beneath the value described in the table then there is a $P>0.05$ chance that such a result could have been due to the presence of an inadequate concentration of gametes and that $\theta$ may still be within the normal range. 


\section{Results}

\section{Estimation of $\mathbf{r}$ and a for 3 separate protocols}

Since the relationship between gamete concentration and penetration rate will depend upon the precise methodology being used to perform the penetration assay, maximum likelihood methods were used to calculate the values of $r$ and $\alpha$ for the distribution of $\theta$ in populations of normal fertile men using 3 different techniques. The values for $\alpha$ and $r$ are given in Table 2 for a conventional type of system with a 7-h preincubation in Medium BWW (Aitken et al., 1982; Rogers, 1985) and 2 modifications with hyperosmotic medium to accelerate capacitation (Aitken et al., 1983) or A23187 to by-pass this process altogether (Aitken et al., 1984b). The observed frequency distribution of hamster oocyte penetration rates obtained with each technique correlated well with the fitted distributions of $\theta$ (Fig. 1), derived from the estimates of $\alpha$ and $r$ presented in Table 2.

Likelihood ratio tests showed that the A23187 system gave a significantly smaller $\alpha$ for a given $r$ (i.e. a greater penetration) than did the conventional $\left(\chi^{2}=23 \cdot 1\right.$, 1d.f., $\left.P<0.001\right)$ or the hyperosmotic $\left(\chi^{2}=30.94\right.$, Id.f., $\left.P<0.001\right)$ systems, although these two systems did not differ significantly $\left(\chi^{2}=2 \cdot 86\right.$, 1d.f.).

Table 2. Estimates of $\alpha$ and $r$ for the distribution of $\theta$ in populations of normal fertile men

\begin{tabular}{lrcc}
\hline Technique & $\alpha$ & $r$ & No. of men \\
\hline Conventional* $^{*}$ & 12.45 & 1.98 & 26 \\
Hyperosmotic $\dagger$ & 4.38 & 1.08 & 42 \\
A23187 & 1.27 & 0.94 & 55 \\
\hline
\end{tabular}

*Aitken et al. (1982).

†Aitken et al. (1983).

$\ddagger$ Aitken et al. (1984b); aqueous suspension of $\mathrm{Ca}^{2+}$ $\mathrm{Mg}^{2+}$ salt at $100 \mu \mathrm{M}(0 \cdot 05 \mathrm{mg} / \mathrm{ml})$.

\section{Estimation of lower limits}

The Poisson-gamma model was applied to each system separately using the methods outlined above and the values of $\alpha$ and $r$ presented in Table 2. The results are presented in Table 3, which gives, for the various values of $t$, the minimum number of gametes $(n x)$ which must be present in the system to identify an individual as having significantly impaired sperm function compared with the normal fertile group. Hence if, in a conventional type of assay, an oligozoospermic sample was found to give a zero penetration score in a system incorporating 20 oocytes and a $1.5 \times 10^{6}$ motile spermatozoa $/ \mathrm{ml}$, reference to Table 3 would indicate that there were insufficient gametes present in the assay to draw any significant conclusions concerning the functional competence of the spermatozoa. Alternatively, if the A23187 system had been used and a sample had given a zero penetration score in an assay system containing 20 oocytes and $1.5 \times 10^{6}$ motile spermatozoa $/ \mathrm{ml}$, then reference to Table 3 would indicate that this result signifies a significant departure in sperm function from the normal population; it could not be explained on the basis of gamete concentration alone. Similarly, if 2 penetrations had been observed following exposure to A23187 in a system incorporating 30 oocytes and $5 \times 10^{6}$ motile spermatozoa $/ \mathrm{ml}$ then a statistically significant $(P<0.05)$ decline in sperm function would again be indicated. However, if only $2.5 \times 10^{6}$ motile spermatozoa $/ \mathrm{ml}$ had been used in this experiment then $n x(30 \times 2.5)$ would have been below the figure of 91 presented in Table 3 and no evidence for a functional deficiency on the part of the spermatozoa would have been indicated. 


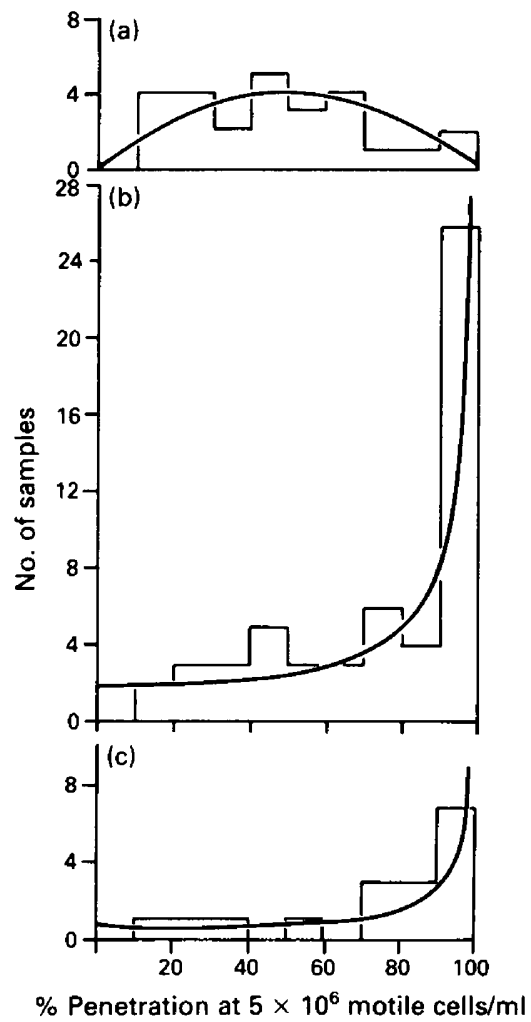

Fig. 1. Relationship between the observed frequency distribution of hamster egg penetration rates and the fitted distributions of $\theta$ based on the estimates of $\alpha$ and $r$ presented in Table 1 and represented as continuous curves. (a) Preincubation for $7 \mathrm{~h}$ with Medium BWW (Aitken et al., 1982); $n=26$; (b) and (c) 3-h preincubation with $100 \mu \mathrm{M}$ and $50 \mu \mathrm{M}$ A23187 respectively (Aitken et al., 1984b), $n=55$ and 17 respectively. Equivalent curves for the hyperosmotic system (Aitken et al., 1983) have been published (Aitken \& Elton, 1986). All penetration assays were carried out using a total sperm concentration of $10 \times 10^{6} / \mathrm{ml}$ and the results were subsequently corrected for a motile sperm concentration of $5 \times 10^{6} / \mathrm{ml}$ using the Poisson model (Aitken \& Elton, 1984).

The low values for the A23187-stimulated system in Table 3 simply indicate that this system is the most sensitive of the protocols examined, requiring significantly fewer spermatozoa to achieve a given level of penetration than the other techniques. The values for the conventional system are slightly lower than with the hyperosmotic technique, largely because of the lower coefficient of variation observed with the former, as indicated by the larger value for $r$ (Table 2).

\section{Discussion}

This study has addressed the quantitative nature of sperm-egg interaction in the zona-free hamster egg assay with particular reference to its application in the analysis of oligozoospermic samples. Difficulties arise in the analysis of such cases because of the high concentrations of spermatozoa required to achieve sperm-oocyte fusion in the penetration assay. Even when using samples from the normal fertile population, at least $2-3 \times 10^{6}$ motile spermatozoa $/ \mathrm{ml}$ and 25 oocytes must be present in the incubation medium in order to be certain that a positive result will be observed with a 
Table 3. Table of limits indicating the gamete concentrations $(n x)^{*}$ which must be exceeded if a given level of penetration is to be indicative of defective sperm function with a probability of $P<0.05$

\begin{tabular}{cccc}
\hline \multirow{2}{*}{$\begin{array}{c}\text { Penetration } \\
\text { result }(t) \dagger\end{array}$} & \multicolumn{3}{c}{ Protocol } \\
\cline { 2 - 4 } & Conventional + & Hyperosmotic§ & A23187ף \\
\hline 0 & 44 & 66 & 29 \\
1 & 81 & 132 & 60 \\
2 & 118 & 197 & 91 \\
3 & 154 & 264 & 122 \\
4 & 190 & & 153 \\
5 & 226 & & 184 \\
6 & & & 215 \\
\hline
\end{tabular}

${ }^{*}$ Gamete concentration $(n x)=$ no. of oocytes $\times$ conc. of motile spermatozoa $\times 10^{6} / \mathrm{ml}$.

$\dagger \mathrm{t}=$ total no. of penetrations $\left(n m_{1}\right)=$ no. of oocytes $\times$ no. of spermatozoa per ovum.

$\ddagger$ Preincubation for $7 \mathrm{~h}$ in Medium BWW (Aitken et al., 1982).

$\S$ Preincubation for $3 \mathrm{~h}$ in hyperosmotic Medium BWW (Aitken et al., 1983).

TPreincubation for $3 \mathrm{~h}$ in A23187 prepared as an aqueous suspension of the $\mathrm{Ca}^{2+} / \mathrm{Mg}^{2+}$ salt at $100 \mu \mathrm{M}(0.05 \mathrm{mg} / \mathrm{ml}$ ) (Aitken et al., 1984b).

conventional type of assay (Table 3). In contrast, fertilization in the homologous human in-vitro fertilization system can be achieved with only $10^{4}-10^{5}$ motile spermatozoa (Mahadevan \& Baker, 1984). This difference is probably due to the ability of the zona pellucida to induce an acrosome reaction in the vicinity of the oocyte in the homologous situation, whereas the penetration assay depends entirely upon the occurrence of spontaneous acrosome reactions in the ambient medium (Singer et al., 1985).

Accelerating the process of capacitation by subjecting the spermatozoa to hyperosmotic conditions shortens the time course of the assay (Aitken et al., 1983) but does not significantly increase its sensitivity (Tables $2 \& 3$ ). In contrast, exposing the spermatozoa to the ionophore, A23187, both shortens the time course of the assay and also increases its sensitivity, such that significantly fewer gametes need be incorporated into the system in order to achieve a given level of penetration (Table 3).

Since the penetration assay only assesses a limited range of sperm functions its diagnostic application focuses on the interpretation of zero or low penetration results. Clearly such results may arise because of a significant decline in the functional competence of the spermatozoa $(\theta)$ or, in cases of oligo- and/or asthenozoospermia, because the concentration of gametes is insufficient to maintain an adequate collision frequency with the oocytes. To some extent this problem may be circumvented by using 'swim up' techniques to isolate and concentrate the motile spermatozoa (Rogers, 1985). However, even with such procedures it is frequently impossible to achieve the desired 2-3 $310^{6}$ motile spermatozoa $/ \mathrm{ml}$ with severely oligoasthenozoospermic samples. Alternatively, formulae based on the Poisson model may be used to correct results to a theoretical constant concentration of motile spermatozoa (Aitken \& Elton, 1984). However, such techniques are not effective in cases of zero penetration because such a result would remain zero even after the above formulae had been applied.

In the present study we have extended the Poisson model to surmount this problem. Using a Poisson-gamma model to obtain an accurate description of the distribution of $\theta$ for populations of normal fertile men (Fig. 1), we have been able to calculate the relationship between gamete concentration and oocyte penetration for 3 separate protocols. For any given level of penetration $(t)$ 
achieved with a specified protocol, the value of $n x$ must be exceeded if the result is to be taken as evidence of a significant $(P<0.05)$ reduction in the functional competence of the spermatozoa, relative to the normal fertile population.

The values described in Table 3 will be of direct relevance to those working with similar systems in evaluating the statistical and biological significance of low or negative results in the penetration assay. While the influence of sperm number on the outcome of the assay has been examined in previous studies (Binor et al., 1980; Aitken et al., 1982), this is the first analysis to recognize the significance of gamete concentration, i.e. the number of oocytes as well as the concentration of spermatozoa, in the incubation medium. In the Poisson-gamma model gamete concentration is represented by the product of oocyte number and motile sperm concentration $(n x)$. This form of presentation (Table 3 ) permits adjustment to be made in the concentration of either gamete in order to achieve the minimal values set out in Table 3 . Hence in cases of oligozoospermia any deficiency in the numbers of spermatozoa can be compensated for by an increase in the number of oocytes introduced into the sperm suspensions. Using the statistical procedures described in this paper it should be possible to create similar tables of limits for any given methodology and thereby provide a rational means of achieving a statistically valid interpretation of the results achieved with this bioassay procedure.

We thank Mr D. W. Richardson and Ms J. S. Clarkson for their invaluable assistance with these studies.

\section{References}

Aitken, R.J. (1985) Diagnostic value of the zona-free hamster oocyte penetration test and sperm movement characteristics in oligozoospermia. Int. J. Androl. 8, 348-356.

Aitken, R.J. \& Elton, R.A (1984) Significance of Poisson distribution theory in analysing the interaction between human spermatozoa and zona free hamster oocytes. J. Reprod. Fert. 72, 311-321.

Aitken, R.J. \& Elton, R.A. (1986) Application of Poisson distribution theory to the zona free hamster oocyte penetration test to assess sperm function of men with asthenozoospermia. J. Reprod. Fert. 77, 67-74.

Aitken, R.J., Best, F.S.M., Richardson, D.W., Djahanbakhch, O. \& Lees, M.M. (1982) The correlates of fertilizing capacity in normal fertile men. Fert. Steril. 38, 68-76.

Aitken, R.J., Wang, Y-F., Lui, J., Best, F.S.M. \& Richardson, D.W. (1983) The influence of medium composition, osmolarity and albumin content on the acrosome reaction and fertilizing capacity of human spermatozoa: development of an improved zona free hamster egg penetration test. Int. J. Androl. 6, 180-193.

Aitken, R.J., Best, F.S.M., Warner, P. \& Templeton, A. (1984a) A prospective study of the relationship between semen quality and fertility in cases of unexplained infertility. J. Androl. 5, 297-303.

Aitken, R.J., Ross, A., Hargreave, T., Richardson, D. \& Best, F. (1984b) Analysis of human sperm function following exposure to the ionophore A23187. $J$. Androl. 5, 321-329.

Biggers, J.D., Whitten, W.K. \& Whittingham, D.G. (1971) The culture of mouse embryos in vitro. In Methods in Mammalian Embryology, pp. 86-116. Ed. J. C. Daniel. Freeman, San Francisco.
Binor, Z., Sokoloski, V.E. \& Wolf, D.P. (1980) Penetration of the zona free hamster egg by human sperm. Fert. Steril. 33, 321-327.

Johnson, N.L. \& Kotz, S. (1969) Distributions in Statistics: Discrete Distributions. Houghton Mifflin, Boston.

Mahadevan, M. \& Baker, G. (1984) Assessment and preparation of semen for in vitro fertilization. In Clinical In Vitro Fertilization, pp. 83-97. Eds C. Wood \& A. Trounson. Springer Verlag, Berlin.

Mather, K. (1964) Statistical Analysis in Biology. Methuen \& Co., Ltd, London.

Rogers, B.J. (1985) The sperm penetration assay: its usefulness re-evaluated. Fert. Steril. 43, 821-840.

Rogers, B.J., van Campen, H., Ueno, M., Lambert, H., Bronson, R. \& Hale, R. (1979) Analysis of human spermatozoa fertilizing ability using zona-free ova. Fert. Steril. 32, 664-670.

Singer, S.L., Lambert, H., Overstreet, J.W., Yanagimachi, R. \& Hanson, F.W. (1985) The kinetics of human sperm binding to the human zona pellucida and zona-free hamster oocyte in vitro. Gamete Res. 12, 29-25.

World Health Organisation (1980) Laboratory Manual for the Examination of Human Semen and SemenCervical Mucus Interaction. Eds M. A. Belsey, R. Eliasson, A. J. Galegos, K. S. Moghissi, C. A. Paulsen \& M. R. N. Prasad. Press Concern, Singapore.

Yanagimachi, R., Yanagimachi, H. \& Rogers, B.J. (1976) The use of zona free animal ova as a test system for the assessment of the fertilizing capacity of human spermatozoa. Biol. Reprod. 15, 471-476.

Received 9 May 1986 